Multi-Objective Optimization for the

National Ignition Facility's Gamma

Reaction History Diagnostic

G. R. Labaria, J. A. Liebman, D. B. Sayre, H. W. Herrmann, E. J. Bond, J. A. Church

January 18, 2013

SPIE Photonics West Conference San Francisco, CA, United States

February 2, 2013 through February 7, 2013 
This document was prepared as an account of work sponsored by an agency of the United States government. Neither the United States government nor Lawrence Livermore National Security, LLC, nor any of their employees makes any warranty, expressed or implied, or assumes any legal liability or responsibility for the accuracy, completeness, or usefulness of any information, apparatus, product, or process disclosed, or represents that its use would not infringe privately owned rights. Reference herein to any specific commercial product, process, or service by trade name, trademark, manufacturer, or otherwise does not necessarily constitute or imply its endorsement, recommendation, or favoring by the United States government or Lawrence Livermore National Security, LLC. The views and opinions of authors expressed herein do not necessarily state or reflect those of the United States government or Lawrence Livermore National Security, LLC, and shall not be used for advertising or product endorsement purposes. 


\title{
Multi-objective optimization for the National Ignition Facility's Gamma Reaction History diagnostic
}

\author{
George R. Labaria ${ }^{a, b}$, Judith A. Liebman ${ }^{b}$, Daniel B. Sayre ${ }^{b}$, Hans W. Herrmann ${ }^{c}$, Essex J. \\ Bond $^{b}$, and Jennifer A. Church ${ }^{b}$ \\ ${ }^{a}$ University of California, Berkeley; Berkeley, California, USA \\ ${ }^{b}$ Lawrence Livermore National Laboratory; Livermore, California, USA \\ ${ }^{c}$ Los Alamos National Laboratory; Los Alamos, New Mexico, USA
}

\begin{abstract}
The National Ignition Facility (NIF) is producing experimental results for the study of Inertial Confinement Fusion (ICF). The Gamma Reaction History (GRH) diagnostic at NIF can detect gamma rays to measure fusion burn parameters such as fusion burn width, bang time, neutron yield, and areal density of the compressed ablator for cryogenic deuterium-tritium (DT) implosions. Gamma-ray signals detected with this diagnostic are inherently distorted by hardware impulse response functions (IRFs) and gains, and are comprised of several components including gamma rays from laser-plasma interactions (LPI). One method for removing hardware distortions to approximate the gamma-ray reaction history is deconvolution. However, deconvolution of the distorted signal to obtain the gamma-ray reaction history and its associated parameters presents an ill-posed inverse problem and does not separate out the source components of the gamma-ray signal. A multi-dimensional parameter space model for the distorted gamma-ray signal has been developed in the literature. To complement a deconvolution, we develop a multi-objective optimization algorithm to determine the model parameters so that the error between the model and the collected gamma-ray data is minimized in the least-squares sense. The implementation of the optimization algorithm must be sufficiently robust to be used in automated production software. To achieve this level of robustness, impulse response signals must be carefully processed and constraints on the parameter space based on theory and experimentation must be implemented to ensure proper convergence of the algorithm. In this paper, we focus on the optimization algorithm's theory and implementation.
\end{abstract}

Keywords: multi-objective, optimization, gamma-ray reaction history

\section{INTRODUCTION}

The National Ignition Facility (NIF) at the Lawrence Livermore National Laboratory (LLNL) is a 192-beam pulsed laser system. It is the world's largest and most powerful laser system in use for the study of Inertial Confinement Fusion and high-energy-density (HED) science. A primary goal of the NIF is to achieve ignition of a deuterium-tritium (DT) fuel capsule and provide access to the study of fusion ignition and the physics of nuclear weapons [1].

The Gamma Reaction History (GRH) diagnostic uses gas Cherenkov detectors to measure gamma-rays in specific energy ranges. The diagnostic contains four identical Cherenkov gas cells with variable thresholds on gamma-ray energy. These are typically configured at 2.9, 4.5, 8, and $10 \mathrm{MeV}$. Each cell contains four scope channels whose output is stitched together by a stitching algorithm providing a recording of the gamma-ray signal. Additional information on the GRH diagnostic and the stitching algorithm can be found in [2] and [3] respectively.

The gamma-ray signals detected by the GRH diagnostic scopes are distorted by hardware impulse response functions (IRFs) and hardware gains. These distortions must be removed in order to obtain useful performance metrics on the DT implosion experiments. The multi-objective optimization algorithm seeks to remove the

Further author information:

George R. Labaria: E-mail: labaria.george@berkeley.edu

Judith A. Liebman: E-mail: liebman1@llnl.gov 


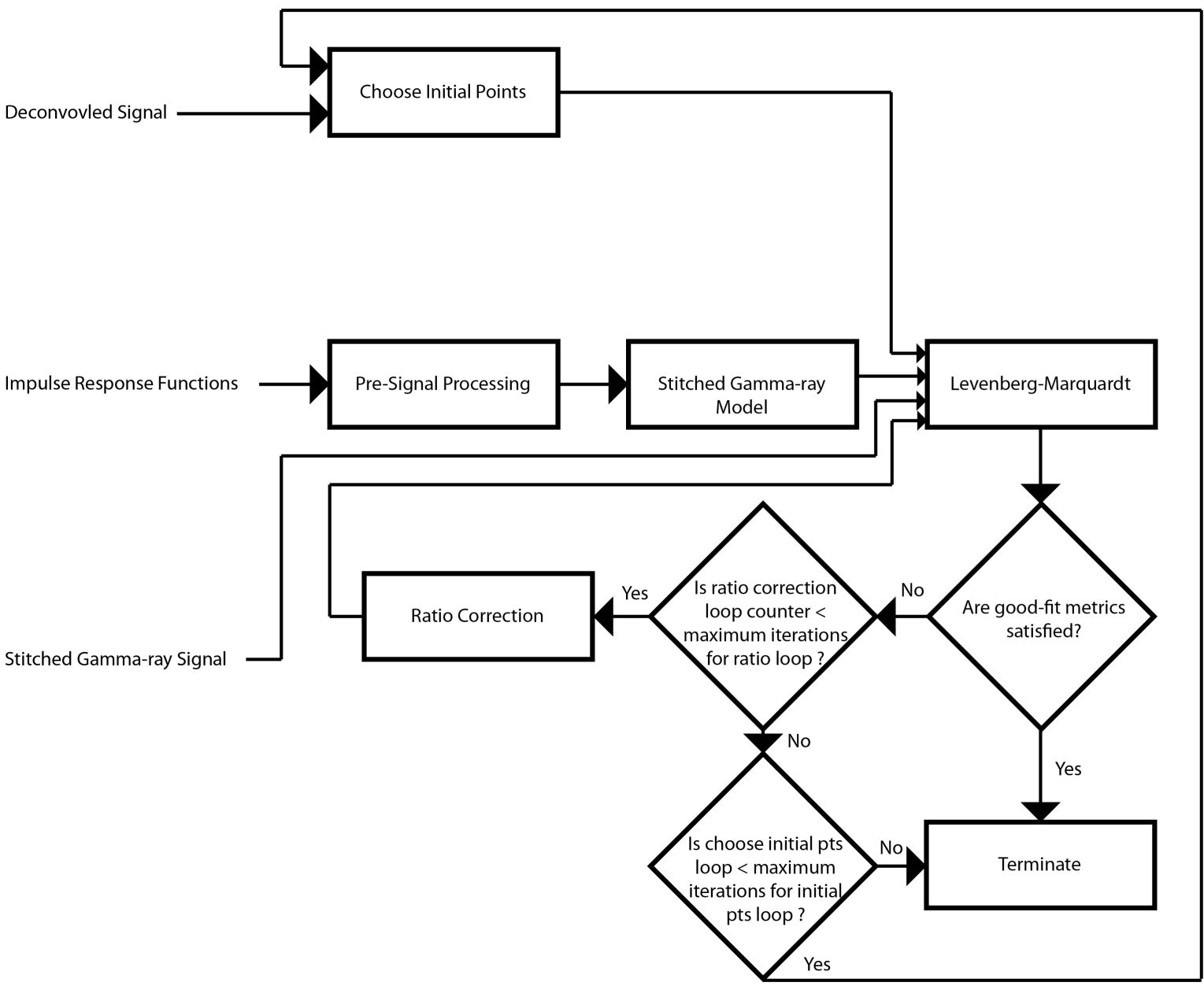

Figure 1. A flow chart of the entire multi-objective algorithm.

hardware impulse response functions and gains as well as estimate the components of the gamma-ray signal. This is accomplished by convolving a model of the gamma-ray signal with the hardware impulse responses that distort it and fitting the gamma-ray model parameters to the stitched gamma-ray signal. A model of the stitched gamma-ray signal and a forward-fit routine has been developed in [4]. We use the stitched gamma-ray model developed in Ref. 4 as our objective function to our optimization algorithm. The core of the optimization makes use of a modified Levenberg-Marquardt algorithm which is derived in detail in [5] and [6]. Special care is taken to restrict the parameter space and process the impulse response functions to ensure proper convergence of the algorithm to local minima that is closest to the objective model function in the sense of least-squares and other metrics which we derive in this paper; this also leads to a more robust optimization algorithm which can be implemented in automated production software. The optimization is done simultaneously to all four scopes stitched gamma-ray signal, making this a multi-objective optimization. A flow chart of the entire multi-objective algorithm is given in figure 1. In the next section and subsequent subsections, we will explain each step of the algorithm (represented by the blocks in figure 1).

\section{ALGORITHM DERIVATION}

In this section we derive our multi-objective optimization algorithm for simultaneously fitting the scopes' stitched gamma-ray signal to the model function developed in [4].

Let $S_{i}(t)$ be the stitched gamma-ray signal for scopes $i=1,2, \ldots, k$. For a typical GRH configuration, four scopes corresponding to Cherenkov gas cells of 2.9, 4.5, 8, and $10 \mathrm{MeV}$ are used, so $k=4$ in this case. The 
stitched gamma-ray model in [4] separates the signal into five components: deuterium-tritium (DT), capsule (CAP, also known as the ablator), hohlraum (HOHL), thermo-mechanical package (TMP), and background from laser-plasma interactions (LPI) within the hohlraum. The following maps defined in Table 1 are used in the stitched gamma-ray model. Note that the symbol " $\otimes$ " is the symbol for binary operation convolution.

Table 1. Stitched Gamma-ray Model Functions

\begin{tabular}{|c|c|}
\hline Function & Description \\
\hline \multirow[t]{2}{*}{$\mathcal{R H}$} & time profile of the gamma-ray reaction history; \\
\hline & modeled by a Gaussian defined by $\left(t, \mu_{R H}, \sigma_{R H}, A_{R H}\right) \mapsto A_{R H} \exp \left(-\frac{1}{2}\left(\frac{t-\mu_{R H}}{\sigma_{R H}}\right)^{2}\right)$ \\
\hline \multirow[t]{2}{*}{$\rho R$} & time profile of the areal density of the compressed ablator; \\
\hline & modeled by a Gaussian defined by $\left(t, \mu_{\rho R}, \sigma_{\rho R}, A_{\rho R}\right) \mapsto A_{\rho R} \exp \left(-\frac{1}{2}\left(\frac{t-\mu_{\rho R}}{\sigma_{\rho R}}\right)^{2}\right)$ \\
\hline$\overline{\mathcal{I R} \mathcal{F}}$ & $\begin{array}{l}\text { time profile of an impulse response function defined by the discrete convolution } \\
\qquad \mathcal{P M T} \otimes \mathcal{G C} \text { where } \mathcal{P M T} \text { is the impulse response function } \\
\text { of the photomultiplier tubes and } \mathcal{G C} \text { is the impulse response function of the Cherenkov gas cells }\end{array}$ \\
\hline $\mathcal{H O H L}$ & time profile of the impulse response function of the hohlraum \\
\hline $\mathcal{T M P}$ & time profile of the impulse response function of the thermo-mechanical package \\
\hline $\mathcal{L P \mathcal { I }}$ & time profile of the laser-plasma interactions \\
\hline
\end{tabular}

The model function that we will use as the objective function in our optimization algorithm is given by

$$
\begin{aligned}
F_{i}(t, \boldsymbol{\beta})= & C_{P M T_{i}} C_{q e_{i}} C_{c h_{i}} C_{\text {ohm }} \alpha_{D T_{i}} N_{D T}\left(\mathcal{R H} \otimes \mathcal{I R \mathcal { F }}(t)+C_{P M T_{i}} \alpha_{C A P_{i}} N_{C A P}[(\mathcal{R H} \cdot \rho R) \otimes \mathcal{I R \mathcal { F }}](t)+\right. \\
& \alpha_{H O H L_{i}} N_{H O H L}(\mathcal{R H} \otimes \mathcal{I} \mathcal{F} \mathcal{F} \otimes \mathcal{H} \mathcal{H} \mathcal{L})(t)+\alpha_{T M P_{i}} N_{T M P}(\mathcal{R H} \otimes \mathcal{I} \mathcal{F} \otimes \mathcal{T} \mathcal{H P})(t)+ \\
& \alpha_{L P I_{i}} N_{L P I_{i}} \mathcal{L P} \mathcal{I}(t)
\end{aligned}
$$

for scopes $i=1,2, \ldots, k$ and where $\boldsymbol{\beta} \in \mathbb{R}^{10+k}$ is the parameter vector,

$$
\boldsymbol{\beta}=\left(\mu_{R H}, \sigma_{R H}, A_{R H}, \mu_{\rho R}, \sigma_{\rho R}, A_{\rho R}, N_{D T}, N_{C A P}, N_{H O H L}, N_{T M P}, N_{L P I_{1}}, N_{L P I_{2}}, N_{L P I_{3}}, N_{L P I_{4}}, \ldots, N_{L P I_{k}}\right)
$$

whose entries are described in Table 2. The variables $\alpha_{D T_{i}}, \alpha_{C A P_{i}}, \alpha_{H O H L_{i}}, \alpha_{T M P_{i}}$, and $\alpha_{L P I_{i}}$ are what we call "ratio factors" which are held fixed during the Levenberg-Marquardt procedure and modified later in the

\begin{tabular}{|c|c|}
\hline Parameter & Description \\
\hline$\mu_{R H}, \sigma_{R H}, A_{R H}$ & $\begin{array}{c}\text { mean, standard deviation, and amplitude of the gamma-ray reaction } \\
\text { history Gaussian, respectively }\end{array}$ \\
\hline$\mu_{\rho R}, \sigma_{\rho R}, A_{\rho R}$ & $\begin{array}{l}\text { mean, standard deviation, and amplitude of the areal } \\
\text { density of the compressed ablator model Gaussian, respectively }\end{array}$ \\
\hline$N_{D T}, N_{C A P}, N_{H O H L}, N_{T M P}$ & $\begin{array}{l}\text { normalization factors for the DT, capsule, hohlraum, and TMP } \\
\text { components respectively }\end{array}$ \\
\hline
\end{tabular}
ratio-correcting stage of our algorithm. See section 2.4 for an in depth discussion on the ratio-correcting scheme. The constants $C_{P M T_{i}}, C_{q e_{i}}, C_{c h_{i}}$, and $C_{o h m}$ are hardware gain factors and will be discussed in section 2.7.

Table 2. Parameter Descriptions

At first glance, the parameter space looks to be quite large. But for a typical four-cell GRH configuration, $k=4$, and hence our parameter space is 14 -dimensional (i.e., $\beta \in \mathbb{R}^{14}$ ). In section 2.5 , we will introduce restrictions on these parameters that will greatly reduce the parameter space further.

In practice, the stitched gamma-ray data and the hardware impulse response functions are sampled at unique times - they are typically not sampled at the same time - giving us discrete values. The differences in the sample 
times of the stitched gamma-ray data and the hardware impulse response functions are problematic in a discrete convolution and will be addressed in section 2.1.

For each scope $i$, let

$$
\begin{aligned}
\mathbf{t}_{i}^{\mathrm{stit}} & =\left(t_{i_{1}}^{\mathrm{stit}}, t_{i_{2}}^{\mathrm{stit}}, \ldots, t_{i_{n_{i}^{\mathrm{stit}}}^{\mathrm{stit}}}\right)^{T} \\
\mathbf{t}_{i}^{\mathrm{pmt}} & =\left(t_{i_{1}}^{\mathrm{pmt}}, t_{i_{2}}^{\mathrm{pmt}}, \ldots, t_{i_{n_{i}^{\mathrm{pmt}}}^{\mathrm{pmt}}}^{\mathrm{pmt}}\right)^{T} \\
\mathbf{t}_{i}^{\mathrm{gc}} & =\left(t_{i_{1}}^{\mathrm{gc}}, t_{i_{2}}^{\mathrm{gc}}, \ldots, t_{i_{n_{i}^{\mathrm{gc}}}^{\mathrm{gc}}}^{\mathrm{gc}}\right)^{T}
\end{aligned}
$$

be the time vectors for the stitched gamma-ray data, PMT and gas cell impulse response functions respectively, and let $T$ denote the matrix transpose. Further, let $\mathbf{t}^{\text {hohl }}=\left(t_{1}^{\text {hohl }}, t_{2}^{\text {hohl }}, \ldots, t_{n^{\text {hohl }}}^{\text {hohl }}\right)^{T}$ and $\mathbf{t}^{\text {tmp }}=$ $\left(t_{1}^{\mathrm{tmp}}, t_{2}^{\mathrm{tmp}}, \ldots, t_{n^{\mathrm{tmp}}}^{\mathrm{tmp}}\right)^{T}$ be the time vectors for the hohlraum and TMP impulse response functions respectively. As previously mentioned, these times are not necessarily the same. Form the following vectors, for each scope $i$,

$$
\begin{aligned}
\mathbf{y}_{i}^{\text {stit }} & =\left(y_{i_{1}}^{\text {stit }}, y_{i_{1}}^{\text {stit }}, \ldots, y_{i_{n_{i}^{\mathrm{stit}}}^{\mathrm{stit}}}\right)^{T} \\
\mathbf{y}_{i}^{\mathrm{pmt}} & =\left(y_{i_{1}}^{\mathrm{pmt}}, y_{i_{2}}^{\mathrm{pmt}}, \ldots, y_{i_{n_{i}^{\mathrm{pmt}}}^{\mathrm{pmt}}}^{\mathrm{pm}}\right)^{T} \\
\mathbf{y}_{i}^{\mathrm{gc}} & =\left(y_{i_{1}}^{\mathrm{gc}}, y_{i_{2}}^{\mathrm{gc}}, \ldots, y_{i_{n_{i}^{\mathrm{gc}}}^{\mathrm{gc}}}^{\mathrm{gc}}\right)^{T}
\end{aligned}
$$

where $y_{i_{j}}^{\text {stit }}=S_{i}\left(t_{i_{j}}^{\text {stit }}\right)$ for $j=1,2, \ldots, n_{i}^{\text {stit }} ; y_{i_{j}}^{\mathrm{pmt}}=\mathcal{P} \mathcal{M T}\left(t_{i_{j}}^{\mathrm{pmt}}\right)$ for $j=1,2, \ldots, n_{i}^{\mathrm{pmt}} ;$ and $y_{i_{j}}^{\mathrm{gc}}=\mathcal{G C}\left(t_{i_{j}}^{\mathrm{gc}}\right)$ for $j=1,2, \ldots, n_{i}^{\mathrm{gc}}$. We construct the $\mathcal{H O H} \mathcal{L}$ and $\mathcal{T} \mathcal{M P}$ impulse response function vectors, $\mathbf{y}^{\text {hohl }}$ and $\mathbf{y}^{\text {tmp }}$ respectively in a similar manner. We are not yet ready to make use of these values. A discrete time convolution between two signals requires the signals to be sampled at the same times. Another problem is that the "start time" of the signals may not coincide, and hence time-shifting of the signals as a result of a discrete time convolution will occur. We make these issues more precise in the next section.

\subsection{Pre-Signals Processing}

We first define the "peak" and "start time" of a signal.

Definition 1. Let $f$ be a discrete signal defined on a set of discrete times $T=\left\{t_{1}, t_{2}, \ldots, t_{n} \mid t_{1}<t_{2}<\cdots<t_{n}\right\}$. Let $G=\left\{(t, y) \in \mathbb{R}^{2} \mid f(t)=y\right\}$ be the graph of $f$, and let $\left(t_{M}, y_{M}\right)$ be a data point such that $y_{M}=\max _{T} f\left(t_{M}\right)$. We define the peak region $P \subset G$ with peak threshold $0<\delta<1$ to be the set of points such that the following hold:

1. $\left(t_{M}, y_{M}\right) \in P$,

2. $\left(t_{M+j}, f\left(t_{M+j}\right)\right) \in P$ if $\left(t_{M+j-1}, f\left(t_{M+j-1}\right)\right) \in P$ and $f\left(t_{M+j}\right) \geq \delta y_{M}$, and

3. $\left(t_{M-j}, f\left(t_{M-j}\right)\right) \in P$ if $\left(t_{M-j+1}, f\left(t_{M-j+1}\right)\right) \in P$ and $f\left(t_{M-j}\right) \geq \delta y_{M}$,

for $j \in \mathbb{N}$.

Throughout the rest of this paper, we will refer to a peak region of a signal defined with a fixed peak threshold $\delta=\delta_{0}$ where $0<\delta_{0}<1$, unless otherwise noted.

Definition 2. The time $t_{0}$ is a start time of a signal if $\left(t_{0}, f\left(t_{0}\right)\right) \in P$ and $t_{0} \leq t_{j}$ for all $j=1,2, \ldots, n$.

Definition 3. Let $f_{1}$ and $f_{2}$ be two signals defined on a set of discrete times $T_{1}=\left\{t_{1_{1}}, t_{1_{2}}, \ldots, t_{1_{n_{1}}} \mid t_{1_{1}}<t_{1_{2}}<\cdots<t_{1_{n_{1}}}\right\}$ and $T_{2}=\left\{t_{2_{1}}, t_{2_{2}}, \ldots, t_{2_{n_{2}}} \mid t_{2_{1}}<t_{2_{2}}<\cdots<t_{2_{n_{2}}}\right\}$ respectively. Also let $P_{1}$ and $P_{2}$ be their respective peak regions. We say that signals $f_{1}$ and $f_{2}$ have the same start times if the following hold: 
1. $\left(t_{1_{1}}, f_{1}\left(t_{1_{1}}\right)\right) \in P_{1},\left(t_{2_{1}}, f_{2}\left(t_{2_{1}}\right)\right) \in P_{2}$, and

2. $t_{1_{1}}$ is the start time of $f_{1}$ and $t_{2_{1}}$ is the start time of $f_{2}$.

Figure 2 gives an example of two pairs of signals, one pair with the same start time and the other with a different start time.

The importance of these three definitions is that it allows us to talk about when exactly the IRF signals "start." The data recorded prior to the peak region is random noise, and in fact is used to calculate the variance of our data when defining the reduced chi-squared statistic (see section 2.2). Thus by removing this extraneous signal data, we can improve the quality of our convolutions. Hence we define the new truncated versions of the IRFs:

Set $\delta_{0}=0.01$, and let $P_{i}^{\mathrm{pmt}}$ and $P_{i}^{\mathrm{gc}}$ be the peak regions with peak threshold $\delta_{0}$ of the PMT and gas cell impulse response functions for scope $i$ respectively; also, let $P^{\text {hohl }}$ and $P^{\mathrm{tmp}}$ be the peak regions with peak threshold $\delta_{0}$ of the hohlraum, and TMP impulse response functions respectively. Let $t_{0_{i}}^{\mathrm{pmt}}, t_{0_{i}}^{\mathrm{gc}}, t_{0}^{\mathrm{hohl}}$, and $t_{0}^{\mathrm{tmp}}$ be their respective start times. Construct the truncated time vectors for each scope $i$ :

$$
\begin{aligned}
\widehat{\mathbf{t}}_{i}^{\mathrm{pmt}} & =\left(t_{i_{k_{i}^{\mathrm{pmt}}}^{\mathrm{pmt}}}^{\mathrm{pm}}, t_{i_{k_{i}^{\mathrm{pmt}}+1}^{\mathrm{pmt}}}^{\mathrm{pmt}}, \ldots, t_{i_{n_{i}^{\mathrm{pmt}}}^{\mathrm{pmt}}}^{\mathrm{pm}}\right)^{T} \\
\widehat{\mathbf{t}}_{i}^{\mathrm{gc}} & =\left(t_{i_{k_{i}^{\mathrm{gc}}}}^{\mathrm{gc}}, t_{i_{k_{i}^{\mathrm{gc}}+1}^{\mathrm{gc}}}^{\mathrm{gc}}, \ldots, t_{i_{n_{i}^{\mathrm{gc}}}^{\mathrm{gc}}}\right)^{T}
\end{aligned}
$$
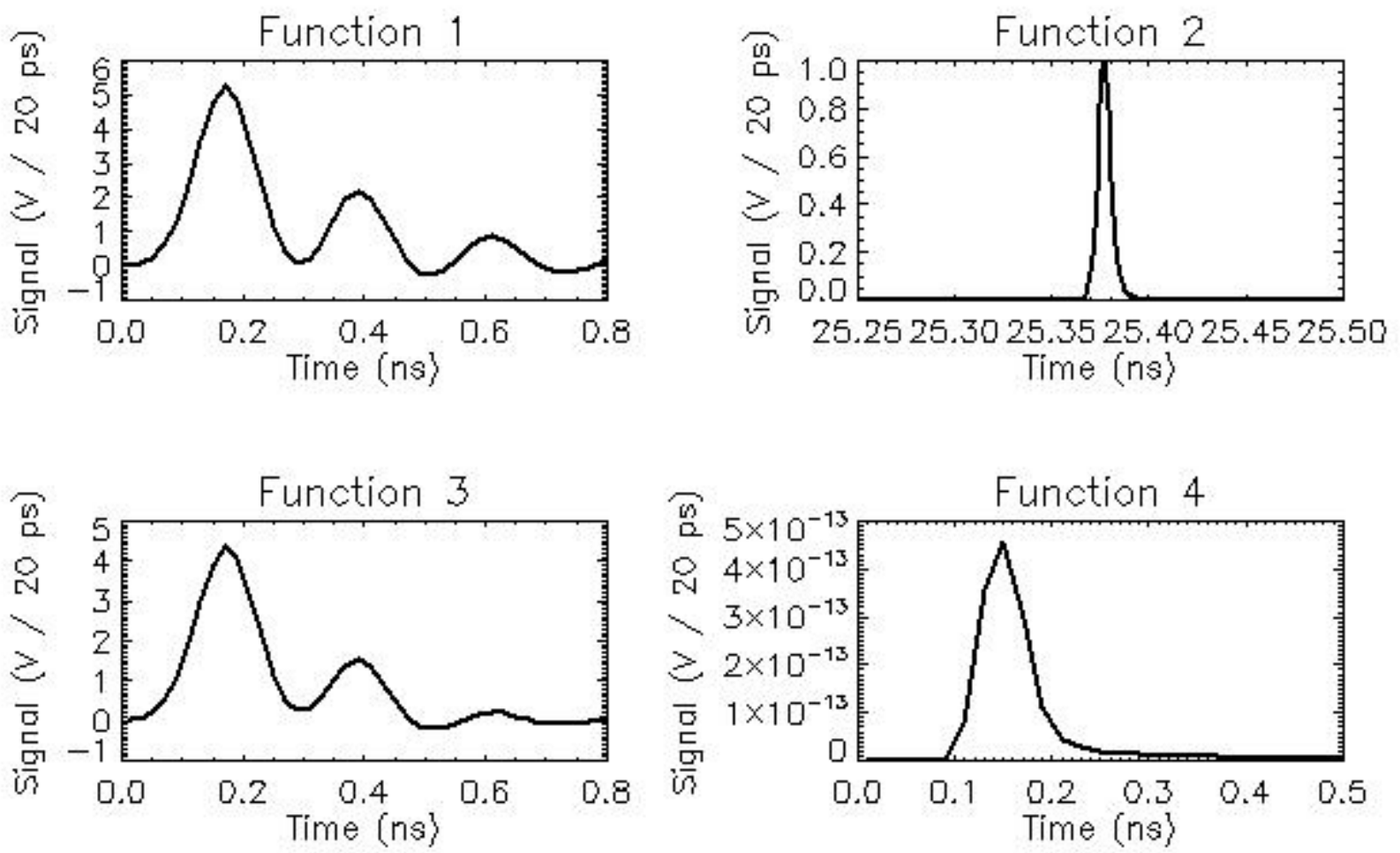

Figure 2. A typical example of impulse response functions. Functions 1 and 3 have the same start time, and functions 2 and 4 have different start times. A discrete time convolution on finite discrete time signals with different start times would cause unwanted time-shifting of the peak region. 
such that $t_{i_{i}^{\mathrm{pmt}}}^{\mathrm{pmt}}=t_{0_{i}}^{\mathrm{pmt}}$ and $t_{i_{k_{i}^{\mathrm{gc}}}^{\mathrm{gc}}}^{\mathrm{gc}}=t_{0_{i}}^{\mathrm{gc}}$. Similarly, let $\widehat{\hat{\mathbf{t}}}^{\text {hohl }}=\left(t_{i_{k_{i}^{\text {hohl }}}}^{\mathrm{hohl}}, t_{i_{k_{i}^{\text {hohl }}+1}^{\text {hohl }}}, \ldots, t_{i_{n_{i}^{\text {hohl }}}}^{\text {hohl }}\right)^{T}$ and $\widehat{\mathbf{t}}^{\mathrm{tmp}}=$ $\left(t_{i_{i} \text { tmp }}^{\mathrm{tmp}}, t_{i_{i}^{\mathrm{tmp}}+1}^{\mathrm{tmp}}, \ldots, t_{i_{i}^{\mathrm{tmp}}}^{\mathrm{tmp}}\right)^{T}$ such that $t_{k_{i}^{\mathrm{hohl}}}^{\mathrm{hohl}}=t_{0}^{\mathrm{hohl}}$ and $t_{k_{i}^{\mathrm{tmp}}}^{\mathrm{tmp}}=t_{0}^{\mathrm{tmp}}$ be the time vectors for the hohlraum and TMP impulse response functions respectively. Form the impulse response vectors $\widehat{\mathbf{y}}^{\mathrm{pmt}}, \widehat{\mathbf{y}}^{\mathrm{gc}}, \widehat{\mathbf{y}}^{\text {hohl }}$ and $\widehat{\mathbf{y}}^{\mathrm{tmp}}$ by sampling the respective impulse response functions at their respective times given by the time vectors.

There is still one modification we must do before we can make use of these impulse response function values. Let $f_{1}$ and $f_{2}$ be two discrete time signals. In order to correctly evaluate the discrete time convolution $f_{1} \otimes f_{2}$, we must ensure that the samples between the two signals coincide in time; that is, they are evaluated at the same times. We make this notion more precise.

Definition 4. Let $f$ be a discrete time signal with time vector $\mathbf{t}=\left(t_{1}, t_{2}, \ldots, t_{n}\right)^{T}$. Define

$$
\Delta t=\min \{\mathbf{t}[i+1]-\mathbf{t}[i] \mid i=1,2, \ldots, n-1\}
$$

to be the time step of $f$. Note that the notation $\mathbf{t}[k]$ means the $k$-th element of the vector $\mathbf{t}$.

Thus let discrete time signals $f_{1}$ and $f_{2}$ have peak times $P_{1}$ and $P_{2}$ respectively, and let $t_{1_{0}}$ and $t_{2_{0}}$ be their respective start times. Further, let $\Delta t_{1}$ and $\Delta t_{2}$ be their respective time steps. Since we will ultimately be performing a discrete time convolution on these two signals, we want to make sure we do not decimate any data; thus we choose the time step that is smallest between these two signals: $\Delta t_{\min }=\min \left\{\Delta t_{1}, \Delta t_{2}\right\}$. Construct new time vectors for $f_{1}$ and $f_{2}$ :

$$
\begin{aligned}
\widetilde{\mathbf{t}}_{1} & =\left(t_{1_{0}}, t_{1_{0}}+\Delta t_{\text {min }}, t_{1_{0}}+2 \Delta t_{\text {min }}, \ldots, t_{1_{0}}+k_{1} \Delta t_{\text {min }}\right)^{T} \\
\widetilde{\mathbf{t}}_{2} & =\left(t_{2_{0}}, t_{2_{0}}+\Delta t_{\text {min }}, t_{2_{0}}+2 \Delta t_{\text {min }}, \ldots, t_{2_{0}}+k_{2} \Delta t_{\text {min }}\right)^{T}
\end{aligned}
$$

where $k_{1}$ and $k_{2}$ are the largest integers such that $k_{1} \leq\left(t_{1_{n_{1}}}-t_{1_{0}}\right) / \Delta t_{\min }$ and $k_{2} \leq\left(t_{2_{n_{2}}}-t_{2_{0}}\right) / \Delta t_{\min }$ respectively. Now it may be the case that the values of signals $f_{1}$ and $f_{2}$ may not be available when evaluated at these new time vectors, so we approximate their values by a cubic spline interpolation at those points.

Thus we choose the minimum time step between the IRFs and interpolate the IRFs if necessary to obtain the values at these new times. After performing these signals processing procedures on the IRF signals, we are now ready to make use of their values in a discrete time convolution. There is one last modification we must do before we move onto the fit algorithm. Since we will eventually be comparing the model function to the stitched gamma-ray signal in our fit, we want to have the same time step as the stitched gamma-ray signal. Thus after performing the necessary convolutions, we interpolate our convolved IRF signals to the stitched gamma-ray signal's time step. A flow chart summarizing the steps in the pre-signals processing stage is given in figure 3.

\subsection{Multi-objective Optimization}

For a typical GRH configuration, four scopes are used at different voltage offsets. Each of these scopes have multiplexed channels to increase the dynamic range of the gamma-ray data recorded in ICF experiments. The stitching algorithm in [3] "stitches" these multiplexed channels together to provide one stitched gamma-ray signal for each of the four scopes. Our algorithm will optimize the model parameters in equation 1 to fit the stitched gamma-ray data in each of the scopes simultaneously, making our optimization multi-objective. The core of our optimization algorithm implements the Levenberg-Marquardt algorithm which will choose optimum parameter values so that the error between the stitched gamma-ray data and the stitched gamma-ray model is minimized in the least-squares sense. To make our objective more clear, consider the general case where we a have a finite set of data points of independent and dependent variables, $\left(x_{i}, y_{i}\right)$. Let $f(x, \boldsymbol{\beta})$ be the model function with parameters $\boldsymbol{\beta}$, and form the vector $\boldsymbol{\Delta}(\boldsymbol{\beta})=\left(y_{1}-f\left(x_{1}, \boldsymbol{\beta}\right), y_{2}-f\left(x_{2}, \boldsymbol{\beta}\right), \ldots, y_{n}-f\left(x_{n}, \boldsymbol{\beta}\right)\right)^{T}$. We wish to minimize the function $\chi^{2}(\boldsymbol{\beta})=\|\Delta(\boldsymbol{\beta})\|^{2}$ using the Levenberg-Marquardt algorithm, where $\|\cdot\|$ refers to the induced $\ell_{2}$ vector norm for operators. The function $\chi^{2}$ is known as the chi-squared error, and division of this function by the number of degrees of freedom gives the reduced chi-squared error. To adapt this optimization problem to our fit, we must define the difference vector $\boldsymbol{\Delta}$ in a way that incorporates the stitched gamma-ray data from all of the 


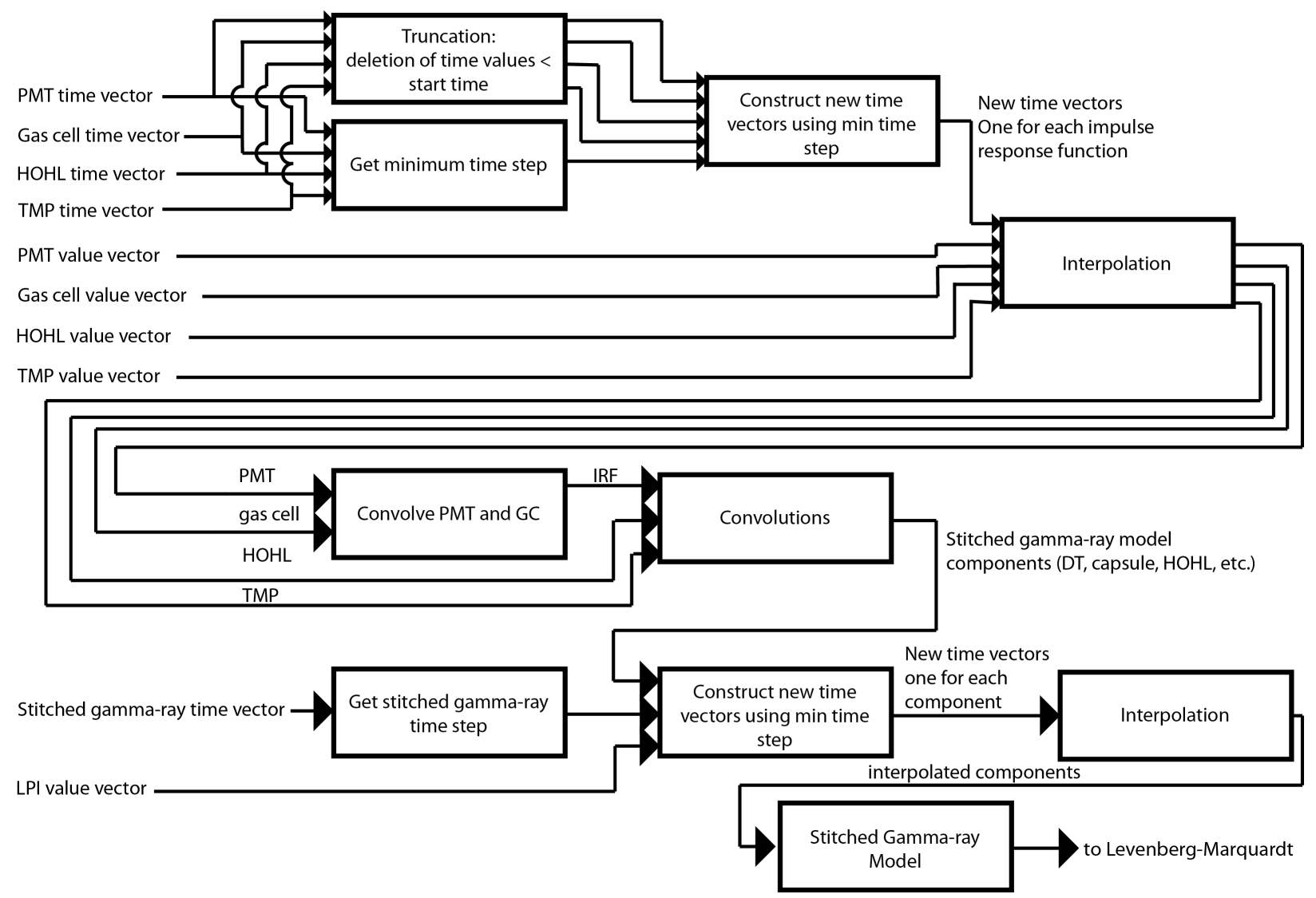

Figure 3. A flow chart of the pre-signals processing step.

scopes so that the parameter values are chosen to simultaneously fit the model functions for each of the scopes' stitched gamma-ray data. Before we define the difference vector, we need one more definition.

Definition 5. Let $f$ be a discrete time signal with graph $G=\left\{\left(t_{1}, f\left(t_{1}\right)\right),\left(t_{2}, f\left(t_{2}\right)\right), \ldots,\left(t_{n}, f\left(t_{n}\right)\right)\right\}$, peak region $P$, and start time $t_{j}, 1 \leq j \leq n$. The noise of the signal $f$ is the subset

$$
\left\{\left(t_{1}, f\left(t_{1}\right)\right),\left(t_{2}, f\left(t_{2}\right)\right), \ldots,\left(t_{j-1}, f\left(t_{j-1}\right)\right)\right\} \subset G .
$$

In other words, the noise of the signal $f$ is all data points that are recorded before the signal's start time.

We are now ready to define the difference vector $\boldsymbol{\Delta}$ which will be used in the least-squares calculation for the Levenberg-Marquardt algorithm. Let $k$ be the number of scopes, and recall that $F_{i}(t, \boldsymbol{\beta})$ is the model function 
for each scope $i$. Define the difference vector $\boldsymbol{\Delta}$ to be the vector-valued function $\boldsymbol{\Delta}: \mathbb{R}^{10+k} \rightarrow \mathbb{R}^{N}$, given by

$$
\boldsymbol{\Delta}(\boldsymbol{\beta})=\left(\begin{array}{c}
\mathbf{y}_{1}^{\text {stit }}[1] / s_{1}^{2} \\
\mathbf{y}_{1}^{\text {stit }}[2] / s_{1}^{2} \\
\vdots \\
\mathbf{y}_{1}^{\text {stit }}\left[n_{1}^{\text {stit }}\right] / s_{1}^{2} \\
\mathbf{y}_{2}^{\text {stit }}[1] / s_{2}^{2} \\
\mathbf{y}_{2}^{\text {stit }}[2] / s_{2}^{2} \\
\vdots \\
\mathbf{y}_{2}^{\text {stit }}\left[n_{2}^{\text {stit }}\right] / s_{2}^{2} \\
\vdots \\
\mathbf{y}_{k}^{\text {stit }}[1] / s_{k}^{2} \\
\mathbf{y}_{k}^{\text {stit }}[2] / s_{k}^{2} \\
\vdots \\
\mathbf{y}_{k}^{\text {stit }}\left[n_{k}^{\text {stit }}\right] / s_{k}^{2}
\end{array}\right)-\left(\begin{array}{c}
F_{1}\left(\mathbf{t}_{1}^{\text {stit }}[1], \boldsymbol{\beta}\right) / s_{1}^{2} \\
F_{1}\left(\mathbf{t}_{1}^{\text {stit }}[2], \boldsymbol{\beta}\right) / s_{1}^{2} \\
\vdots \\
F_{1}\left(\mathbf{t}_{1}^{\text {stit }}\left[n_{1}^{\text {stit }}\right], \boldsymbol{\beta}\right) / s_{1}^{2} \\
F_{2}\left(\mathbf{t}_{2}^{\text {stit }}[1], \boldsymbol{\beta}\right) / s_{2}^{2} \\
F_{2}\left(\mathbf{t}_{2}^{\text {stit }}[2], \boldsymbol{\beta}\right) / s_{2}^{2} \\
\vdots \\
F_{2}\left(\mathbf{t}_{2}^{\text {stit }}\left[n_{2}^{\text {stit }}\right], \boldsymbol{\beta}\right) / s_{2}^{2} \\
\vdots \\
F_{k}\left(\mathbf{t}_{k}^{\text {stit }}[1], \boldsymbol{\beta}\right) / s_{k}^{2} \\
F_{k}\left(\mathbf{t}_{k}^{\text {stit }}[2], \boldsymbol{\beta}\right) / s_{k}^{2} \\
\vdots \\
F_{k}\left(\mathbf{t}_{k}^{\text {stit }}\left[n_{k}^{\text {stit }}\right], \boldsymbol{\beta}\right) / s_{k}^{2}
\end{array}\right),
$$

where $N=n_{1}^{\text {stit }}+n_{2}^{\text {stit }}+\cdots+n_{k}^{\text {stit }}$, and $s_{i}^{2}$ is the sample variance of the noise of the stitched gamma-ray data from scope $i$; that is, suppose we have the stitched gamma-ray data for scope $i$ with peak region $P_{i}$ and start time $t_{i_{i}} ;$ then

$$
s_{i}^{2}=\frac{1}{j_{i}-1} \sum_{m=1}^{n_{i}^{\text {stit }}}\left(\mathbf{y}_{i}^{\text {stit }}[m]\right)^{2}-\frac{j_{i}}{j_{i}-1} \overline{\mathbf{y}}_{i}^{\text {stit }}
$$

is the sample variance of the noise of the stitched gamma-ray data from scope $i$. Here, the notation $\overline{\mathbf{y}}_{i}^{\text {stit }}$ means the arithmetic mean of the entries in the vector $\mathbf{y}_{i}^{\text {stit }}$. Hence we see that the difference vector $\boldsymbol{\Delta}$ is the concatenation of the difference between each of the scopes' stitched gamma-ray data and the gamma-ray model values, divided by the sample variance of the noise. The division by the sample variance of the noise gives the chi-squared error a bit more statistical information about the quality of the fit, but does not actually change the maxima and minima of the chi-squared error function (hence not affecting the optimization results). We thus have the reduced chi-squared error function

$$
\chi^{2}(\boldsymbol{\beta})=\frac{1}{M}\|\boldsymbol{\Delta}(\boldsymbol{\beta})\|^{2}
$$

where $M$ is the number of degrees of freedom; that is, the number of elements in the difference vector $\boldsymbol{\Delta}$ minus the number of elements in the parameter vector $\boldsymbol{\beta}$. We will use the Levenberg-Marquardt algorithm to minimize equation 11.

It is usually the case that the chi-squared error function has many local minima, but we desire the smallest minimum (or global minimum). However, the initial points for the Levenberg-Marquardt algorithm must be sufficiently close to the global minimum in order to achieve the best result (see [5] and [6] for an in depth discussion about the Levenberg-Marquardt algorithm and its convergence properties). Thus the quality of the fit is dependent on the quality of the initial point chosen. We have observed by running our algorithm on many cryogenic DT shots that it is very sensitive to the placement of these initial points. Thus in order to make our algorithm more robust, we only change our initial point choice only if we know for sure that the initial point chosen converges to an undesired local minima and ratio-correction fails to improve the fit to a satisfactory level. The key to knowing whether or not to change our initial point choice is to determine whether or not the resulting fit is satisfactory or poor in automation. This can be accomplished with the help of a few metrics which aim to capture the behavior of curves.

\subsection{Good-fit Metrics}

Our algorithm must determine when a fit is a "good fit" in automation. Although the chi-squared error is a good metric, we find that it is not sufficient in determining satisfactory fits in automation. 
Definition 6. Let $S_{i}$ be the stitched gamma-ray data from scope $i$ for which we have a satisfactory fit for, and let $f$ be the satisfactory fit. Further, let $T_{1}$ and $T_{2}$ be the set of discrete times for which $S_{i}$ and $f$ are defined on respectively. Finally suppose that $S_{i}$ came from cryogenic DT shot $X$. Set

$$
\begin{aligned}
\delta_{\max } & =\left|\max _{T_{1}} S_{i}-\max _{T_{2}} f\right|, \\
\delta_{\text {area }} & =\left|\int_{T_{1}} S_{i}-\int_{T_{2}} f\right|, \\
\delta_{\text {length }} & =\left|L_{T_{1}}\left(S_{i}\right)-L_{T_{2}}(f)\right|, \text { and } \\
\delta_{\text {time }} & =\left|B\left(S_{i}\right)-B(f)\right|,
\end{aligned}
$$

where $L_{\Omega}(f)$ is the arc length of $f$ over $\Omega$, and $B(f)$ is the signal bang time of $f$; i.e., a time $t$ such that $f(t)=\max _{\Omega} f$ (Note that since our signals are discrete, interpolation is necessary to calculate the integral and arc lengths of the signals). Suppose there is another DT shot $Y$ for which we wish to obtain a satisfactory fit for using our algorithm. Let $S_{i}^{\prime}$ and $f^{\prime}$ be the stitched gamma-ray data from scope $i$ and the fit in question associated to shot $Y$. We say that $f^{\prime}$ is a good fit to $S_{i}^{\prime}$ if all of the following hold:

$$
\begin{aligned}
\left|\max _{T_{1}^{\prime}} S_{i}^{\prime}-\max _{T_{2}^{\prime}} f^{\prime}\right| & \leq \delta_{\max }, \\
\left|\int_{T_{1}^{\prime}} S_{i}^{\prime}-\int_{T_{2}^{\prime}} f^{\prime}\right| & \leq \delta_{\text {area }}, \\
\left|L_{T_{1}^{\prime}}\left(S_{i}^{\prime}\right)-L_{T_{2}^{\prime}}\left(f^{\prime}\right)\right| & \leq \delta_{\text {length }}, \text { and } \\
\left|B\left(S_{i}^{\prime}\right)-B\left(f^{\prime}\right)\right| & \leq \delta_{\text {time }} .
\end{aligned}
$$

In order to determine whether we have a good fit or not, we must have a satisfactory fit to compare to. At first this seems a bit circular, but in practice it is not hard to obtain a satisfactory fit for high neutron yield DT shots; these typically serve as good baselines for which to calculate the "good fit metrics" defined in Definition 6.

\subsection{Ratio Correction}

Now that we have defined what it means to have a good fit for the stitched gamma-ray data, we will introduce the ratio-correction scheme of our algorithm which improves the fits in the case that the good fit metrics are not immediately satisfied. The ratio correcting scheme assumes that the time profile of the stitched gamma-ray data and the gamma-ray model components are proportional from shot-to-shot. Just like in our definition of a good fit in the previous section, we need a satisfactory fit to act as a baseline. Let $S_{i}$ be the stitched gamma-ray for scope $i$ from DT shot $X$, and let $c$ be a component signal of the stitched gamma-ray model (DT, capsule, hohlraum, TMP, or LPI). Suppose that $c$ is a component of a satisfactory fit for $S_{i}$. Set

$$
\alpha_{c}=\frac{\max c}{\max S_{i}}
$$

which we call the control ratio. Now suppose that we have another DT shot $Y$ with associated stitched gammaray data $S_{i}^{\prime}$ for scope $i$ with component signal $c^{\prime}$ ( $c^{\prime}$ is of the same type as $c$; that is, if $c$ is the DT component of shot $X$, then $c^{\prime}$ is the DT component of shot $Y$, etc) of the stitched gamma-ray model fit that we want to improve. We want to find the scaling factor $\xi \in \mathbb{R}$ such that $\left(\xi \max c^{\prime}\right) / \max S_{i}^{\prime}=\alpha_{c}$. Thus we have

$$
\xi=\frac{\alpha_{c} \max S_{i}^{\prime}}{\max c^{\prime}}
$$

as our new ratio for component $c^{\prime}$ of the stitched gamma-ray model for shot $Y$; that is $\alpha_{c}=\xi$ in equation 1 where the subscript $c$ is the string "DT", "CAP", "HOHL", "TMP", or "LPI". 


\subsection{Restricting the Parameter Space}

As mentioned in section 2 , the parameter vector $\boldsymbol{\beta}$ has $10+k$ entries where $k$ is the number of scopes. For a typical four-cell GRH configuration we have $k=4$, so our parameter space is $\mathbb{R}^{14}$. Typically, for high neutron yield DT shots, the LPI component of the gamma-ray signal in the 2.9 and $4.5 \mathrm{MeV}$ threshold scopes are negligible; hence we can set those LPI components identically equal to 0 ; that is $N_{L P I_{i_{1}}}=N_{L P I_{i_{2}}}=0$ where $i_{1}$ and $i_{2}$ are the 2.9 and $4.5 \mathrm{MeV}$ scopes.

Another restriction we can impose is that the normalization factors $N_{H O H L}$ and $N_{T M P}$ are proportional, so we can effectively eliminate one of the variables. This brings our parameter space to $\mathbb{R}^{11}$ for a typical four-scope configuration.

To make our algorithm more efficient and robust, it is necessary to determine the high and low neutron yield shots (so we can determine if we can eliminate the low threshold MeV LPI components). This can often be found indirectly by computing the signal-to-noise ratio. Recalling Definition 5 for the noise of a signal, we can compute the signal-to-noise ratio by computing the ratio of the root-mean-square (RMS) of the entire signal to the RMS of the noise. Low neutron yield shots tend to have a lower signal-to-noise ratio than high neutron yield shots.

\subsection{Choosing Initial Points for the Levenberg-Marquardt Algorithm}

A large part of our strategy for choosing initial points rely on the fact that similar types of DT shots are similar to one another; that is, high neutron yield shots are similar in time profile to other high neutron yield shots; low neutron yield shots are similar in time profile to other low neutron yield shots. We can immediately choose an initial point for the reaction history Gaussian parameters $\mu_{R H}, \sigma_{R H}$ and $A_{R H}$ through a deconvolution analysis which can accurately extract these parameters. The remaining parameters can be initialized by taking their corresponding values from previous fits that were satisfactory. Usually this provides reasonable starting points for the Levenberg-Marquardt algorithm since the similarities between of the time profile between shots imply that the chi-squared error functions are also similar. When choosing new initial points in the case that the maximum iteration for ratio-correction loop has been reached, the new initial points can be chosen from other satisfactory fits; by varying the original initial points by a small factor by a random constant; or a combination of the two.

\subsection{Hardware Gains}

Our fit also has to account for the hardware gains which distort the gamma-ray signal. All of the hardware gains are scope dependent with the exception of $C_{o h m}$ which accounts for the effects of the resistance caused by the Mach-Zehnder fiber-optic cable in the GRH diagnostic. See [7] for more information on the Mach-Zehnder fiber-optic cable for the GRH diagnostic. The gain $C_{q e}$ is the quantum efficiency, and $C_{P M T}$ is the hardware gain resulting from the photomultiplier tubes. We also need to take into account the different $\mathrm{MeV}$ thresholds in the Cherenkov gas cells. Let $\Phi_{i}$ be the Cherenkov response function (note that this is different from the Cherenkov gas cell impulse response function $\mathcal{G C}$ introduced in section 2) and $\Gamma$ be the DT gamma-ray spectrum. The Cherenkov gain for scope $i$ with respect to the $8 \mathrm{MeV}$ threshold is defined to be $C_{c h_{i}}=\frac{\Phi_{i} \cdot \Gamma}{\Phi_{i_{8}} \cdot \Gamma}$ where $\Phi_{i_{8}}$ is the Cherenkov response function corresponding to the $8 \mathrm{MeV}$ threshold scope.

\section{APPLICATION TO GAMMA REACTION HISTORY DATA}

Figure 4 displays the result of the multi-objective algorithm ran on cryogenic DT implosion shot N120417. The optimized parameter vector is given by

$$
\boldsymbol{\beta}=\left(22.81,0.063,89317.58,22.93,0.10,97792.21,0.90,4.30 \times 10^{-12}, 1.14 \times 10^{-8}, 1.01,0.00,0.00,0.31\right)^{T} .
$$

From this we can immediately extract some performance metrics on the DT shot, in particular the reaction history and areal density bang times and burn widths. The DT yield can also be extracted from this data [4]. The data from $\boldsymbol{\beta}$ can also be used to draw conclusions about the different components of the gamma reaction history. Table 3 shows a comparison between the extracted metrics from the multi-objective algorithm and the calibrated metrics obtained from a deconvolution analysis for shot N120417. We see from this that the fit shows agreement with previous analysis on this shot. 

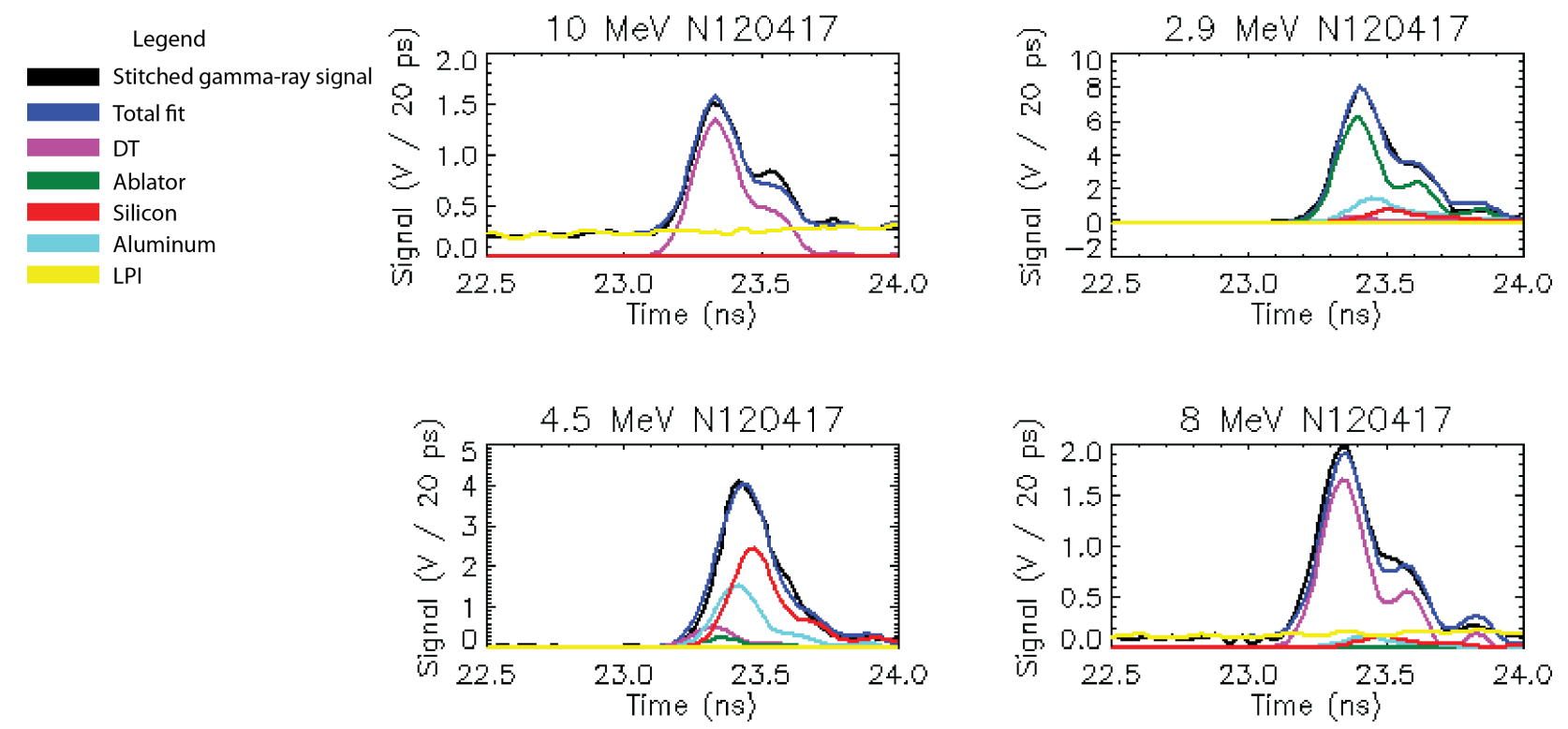

Figure 4. A sample fit to a cryogenic DT shot (N120417). Each panel displays the total fit from different gas cell thresholds with its additive components and the stitched gamma-ray signal for reference.

Table 3. Comparison Between Extracted Metrics from the Multi-objective Algorithm and Calibrated Metrics for Shot N120417's DT and Ablator Components

\begin{tabular}{|c|c|c|c|c|c|c|}
\hline \hline \multicolumn{3}{|c|}{ Extracted Metrics } & \multicolumn{3}{c|}{ Calibrated Metrics } \\
\hline \hline $\begin{array}{c}\text { DT Bang } \\
\text { Time (ns) }\end{array}$ & $\begin{array}{c}\text { DT Burn } \\
\text { Width (ns) }\end{array}$ & $\begin{array}{c}\text { Burn-weighted } \rho R \\
\text { Peak Time (ns) }\end{array}$ & $\begin{array}{c}\text { Burn-weighted } \rho R \\
\text { Peak Width (ns) }\end{array}$ & Scope & $\begin{array}{c}\text { DT Peak } \\
\text { Time (ns) }\end{array}$ & $\begin{array}{c}\text { DT Peak } \\
\text { Width (ns) }\end{array}$ \\
\hline 22.81 & 0.15 & 22.93 & 0.25 & $10 \mathrm{MeV}$ & 22.83 & 0.17 \\
\hline & & & & $2.9 \mathrm{MeV}$ & 22.89 & 0.18 \\
\cline { 5 - 7 } & & & & $4.5 \mathrm{MeV}$ & 22.90 & 0.21 \\
\cline { 5 - 7 } & & & & $8 \mathrm{MeV}$ & 22.82 & 0.16 \\
\hline
\end{tabular}




\section{CONCLUSION AND SUMMARY}

The GRH diagnostic has the ability to determine DT fusion burn width, bang time, yield, and areal density of the compressed ablator in Cryogenic DT shots. A multi-objective fitting routine has been developed to obtain optimized parameters for a best-fit model curve to the GRH stitched gamma-ray signal. Using these parameters, the Gamma Reaction History Gaussian as well as the components of the gamma ray signal are reconstructed. The success or failure of a fit is evaluated by measuring the least-squares error and other "good-fit" metrics which are sufficiently robust to be used in automated production software. Fusion burn parameter results have been compared against the deconvolution results for peak time and peak width and show good agreement.

\section{ACKNOWLEDGMENTS}

We thank the members of the GRH team for their useful discussions. This work was performed under the auspices of the U.S. Department of Energy by Lawrence Livermore National Laboratory under Contract DEAC52-07NA27344.

\section{REFERENCES}

[1] Moses, E. I., "Ignition on the National Ignition Facility: a path towards inertial fusion energy," J. Nucl. Fusion 49(104022), 9pp (2009).

[2] H. W. Herrmann, e. a., "ICF Gamma-ray Reaction History Diagnostics," in [J. Phys.: Conference Series 244], 4pp, The Sixth International Conference on Inertial Fusion Sciences and Applications, IOP Publishing (September 2010). Number: 032047.

[3] C. G. Brown, e. a., "Adaptation of a cubic smoothing spline algorithm for multi-channel data stitching at the National Ignition Facility," in [Proc. of SPIE Vol. 7916], 8pp, High Power Lasers for Fusion Research, SPIE (2011).

[4] D. B. Sayre, e. a., "Multi-shot Analysis of the Gamma Reaction History Diagnostic," in [Review of Scientific Instruments], 3pp, 19-th Topical Conference on High-Temperature Plasma Diagnostics, American Institute of Physics (2012).

[5] Moré, J. J., "The Levenberg-Marquardt Algorithm: Implementation and Theory," in [Numerical Analysis: Proceedings of the Biennial Conference Held at Dundee, June 28-July 1, 1977], 105-116, Springer Berlin Heidelberg (1978). Volume: 630, Number: 0075-8434.

[6] Pujo, J., "The solution of nonlinear inverse problems and the Levenberg-Marquardt method," Geophysics 72(4), 16pp (2007).

[7] E. K. Miller, e. a., "Mach-Zehnder fiber-optic Links for Reaction History Measurements at the National Ignition Facility," in [J. Phys.: Conference Series 244], 4pp, The Sixth International Conference on Inertial Fusion Sciences and Applications, IOP Publishing (September 2010). Number: 032055. 